\title{
Methodology for rapid assessment of blood lithium levels in ultramicro volumes of blood plasma for applications in personal monitoring of patients with bipolar mood disorder
}

\author{
Meha Qassem \\ Iasonas Triantis \\ Michelle Hickey \\ Eleni Palazidou \\ Panayiotis Kyriacou
}




\title{
Methodology for rapid assessment of blood lithium levels in ultramicro volumes of blood plasma for applications in personal monitoring of patients with bipolar mood disorder
}

\author{
Meha Qassem, ${ }^{\mathrm{a}, \star}$ lasonas Triantis, ${ }^{\mathrm{a}}$ Michelle Hickey, ${ }^{\mathrm{a}}$ Eleni Palazidou, ${ }^{\mathrm{b}}$ and Panayiotis Kyriacou ${ }^{\mathrm{a}}$ \\ ${ }^{a}$ City University of London, Research Centre for Biomedical Engineering, London, United Kingdom \\ ${ }^{b}$ Queen Mary University of London, Barts and The London School of Medicine and Dentistry, Whitechapel, London, United Kingdom
}

\begin{abstract}
Bipolar disorder (BD) is a common mental health condition, characterized by extreme changes in mood, energy, and behavior. BD is often managed through mood-stabilizing medications, of which lithium formulations remain the most reliable and effective at reducing the risk of suicide. To achieve adequate and consistent efficacy, lithium concentrations need to be maintained within a narrow therapeutic range $(0.4$ to $1.2 \mathrm{mmol} / \mathrm{L}$ ). Because of its narrow therapeutic index, long-term lithium therapy is associated with serious side effects and risks of toxicity. It is believed that the availability of a personal blood lithium analyzer would benefit patients who are on lithium treatment. We detail the results of a spectrophotometric method performed on ultramicro volumes to determine blood plasma lithium concentrations as compared with reference measurements of flame photometry, and validated in samples of unknown lithium content. Applying multiple linear regression, lithium concentrations could be determined in a rapid manner using full-range spectra or triwavelength data. Both techniques highly correlated with reference standards and could predict lithium levels accurately $\left(R^{2}=0.794214\right.$ and RMSEP $=0.209584$, and $R^{2}=0.863921$ and $\mathrm{RMSEP}=0.167524$, respectively $)$. Therefore, this method can be a useful for rapid assessment of blood lithium in nonlaboratory settings i.e., general practices, hospital clinics, and community health centers by healthcare professionals and/or by patients. Future work will now focus on completion of a miniaturized and integrated system that will deliver a portable and personal lithium-monitoring device. ( 92018 Society of Photo-Optical Instrumentation Engineers (SPIE) [DOI: 10.1117/1.JBO.23.10.107004] Keywords: spectrophotometry; mental health; bipolar disorder; lithium medication; optical sensors.

Paper 180070R received Feb. 2, 2018; accepted for publication Sep. 14, 2018; published online Oct. 24, 2018.
\end{abstract}

\section{Introduction}

Bipolar disorder (BD) is a lifelong mental illness characterized by recurrent episodes of hypomania/mania, depression, and mixed affective states, which causes significant functional impairment. ${ }^{1,2}$ In the UK, the prevalence of BD has been estimated at $\sim 1 \%$ of the population, ${ }^{3}$ costing the National Health Service (NHS) a total of $£ 342$ million in the year 2009/ $2010 .{ }^{4}$ Lithium medication has been widely used to prevent relapse or recurrence of $\mathrm{BP}^{5}$ and it is recommended as "first line" treatment by The National Institute for Health and Care Excellence (NICE) in September 2014. ${ }^{5}$ To be effective, lithium concentrations need to reach a therapeutic level, which ranges between 0.4 and $1.2 \mathrm{mmol} / \mathrm{L}$. Unfortunately, lithium has a narrow therapeutic index, and toxic effects are apparent at concentrations of $\geq 1.4 \mathrm{mmol} / \mathrm{L}$, which can include renal failure, seizures, coma, and even death. ${ }^{6}$ Toxicity can occur suddenly as a result of rapid buildup of lithium concentrations (overdose, drug interactions, and physical illness) or due to cumulative high levels during ongoing chronic therapy. ${ }^{6}$

Because of these limitations, lithium blood levels need to be monitored regularly in patients treated with lithium to ensure optimum efficacy and adequate tolerability. ${ }^{7}$ This is currently done through trimonthly attendance to clinics, where a blood

*Address all correspondence to: Meha Qassem, E-mail: meha.qassem.1@ city .ac.uk sample is taken and sent to the laboratory for analysis using the standard technique of flame emission photometry (FEP). Nevertheless, levels of treatment nonadherence are high and the quality of contemporary lithium monitoring falls short of NICE guidelines. ${ }^{8-10}$ This has resulted in growing demand for both faster, more efficient methods for determining lithium levels, in both clinical settings and at home. Standard techniques for blood lithium measurement such as FEP or atomic absorption spectroscopy (AAS) are not suitable for such applications because they are laboratory-based, cumbersome, expensive, and complex to operate. ${ }^{11}$ Other techniques that have been adapted to suit clinical and home diagnostics include electrophoresis, ${ }^{12,13}$ ion-selective electrodes, ${ }^{11,14,15}$ and impedance spectroscopy. ${ }^{16}$

Ion-selective electrodes can be used for point-of-care testing for determining a range of parameters. The test can involve manual handling that can introduce error, and like impedance spectroscopy, interference with other ions is a possibility even with the recent development of new fluoroionophore (optical sensor), ${ }^{14,17}$ which compromises the specificity and accuracy of the measurement. ${ }^{18}$ As for electrophoresis, lab-on-chip devices based on this technique have been implemented for lithium monitoring, and similar to ion-selective electrodes, the technique can assess various blood components in addition to lithium, but minor variations in $\mathrm{pH}$ can affect charge and flow of molecules, ${ }^{19,20}$ thus measurements must be carried out under

$1083-3668 / 2018 / \$ 25.00$ @ 2018 SPIE 
controlled conditions. Reproducibility is another limitation associated with this technique. ${ }^{20}$

Several studies using optical techniques such as fluorimetry ${ }^{21-23}$ and spectrophotometry ${ }^{24,25}$ have also been employed in blood lithium measurement. A number of these investigated the use of Quinizarin as a reagent to determine lithium levels in pharmaceutical formulations, ${ }^{22,24}$ blood serum, ${ }^{24}$ and saliva. ${ }^{23}$ However, the techniques were developed for laboratory use, i.e., to replace current standard methods, or for batch testing, while simplified versions performed measurements on saliva, which is not a reliable vehicle for clinically acceptable patient monitoring.

There is a need for a technique that can provide rapid measurement of blood lithium in clinical settings, such as general practice (GP) services, clinics, and at home in a simple and accurate manner, with the aim of improving quality of care and outcomes for bipolar patients by adding to the compliance of lithium treatment and reducing the possibility of toxicity. This paper details the result of a spectrophotometric method for measurement of blood lithium that has been optimized for the analysis of ultramicro volumes of sample and investigates the use of a triwavelength system as a method for simplification and miniaturization of the system.

\section{Material and Methods}

Ethics approval was granted by the Senate Research Ethics committee at City University of London prior to performing tests on samples of human blood plasma.

\subsection{Reagents and Materials}

All experiments were performed with analytical grade reagents, and aqueous solutions were prepared using deionized water. A standard solution of $60 \mathrm{mEq} / \mathrm{L}$ lithium carbonate in saline was prepared by dissolving $221.7 \mathrm{mg}$ of lithium carbonate in $100 \mathrm{~mL}$ of water. Subsequently, this was further diluted to make a set of solutions with the following concentrations: 18, 24, 30, 36, 42, 48, and $54 \mathrm{mEq} / \mathrm{L}$. Additional reagents that were prepared are: $0.1 \mathrm{M}$ of $\mathrm{NaOH}, 0.25 \mathrm{M}$ of $\mathrm{Na}_{2} \mathrm{CO}_{3}, 90 \%\left(\mathrm{CH}_{3}\right)_{2} \mathrm{~S}$ (DMSO), and $1 \mathrm{mM}$ of Quinizarin in $\left(\mathrm{CH}_{3}\right)_{2} \mathrm{SO}$.

A set of eight solutions of lithium $\mathrm{Li}_{2} \mathrm{CO}_{3}$ was prepared in saline, with a concentration range of 0.6 to $2.0 \mathrm{mEq} / \mathrm{L}$ in steps of $0.2 \mathrm{mEq} / \mathrm{L}$. This was carried out by diluting a standard solution of $10 \mathrm{mEq} / \mathrm{L}$ of $\mathrm{Li}_{2} \mathrm{CO}_{3}$. Spiked solutions of blood plasma were prepared using mixed pool blood plasma that was acquired from healthy individuals and obtained from a reliable provider (TCS Biosciences Ltd., Buckingham, UK). Samples of human plasma that have been spiked with lithium were prepared by mixing $1 \mathrm{~mL}$ from each reagent of lithium carbonate prepared earlier, i.e., 18, 24, 30, 36, 42, 48, 54, and $60 \mathrm{mEq} / \mathrm{L}$ with $29 \mathrm{~mL}$ of blood plasma, thus giving eight samples of $30 \mathrm{~mL}$, with lithium concentrations of 0.6 to $2.0 \mathrm{mEq} / \mathrm{L}$. Thus, a total of two sets of solutions were prepared with lithium concentration ranging between 0.6 and $2.0 \mathrm{mmol} / \mathrm{L}$ in saline or in blood plasma.

Mixtures of the lithium carbonate solutions made earlier (18 to $60 \mathrm{mEq} / \mathrm{L}$ ) were used to prepare an additional set of six blood plasma samples with unknown amounts of lithium.

\subsection{Testing Procedure}

Saline-based solutions were prepared similarly to the procedure outlined by Gracia et al., ${ }^{24}$ where an amount of $500 \mu \mathrm{L}$ from
$\mathrm{Li}_{2} \mathrm{CO}_{3} /$ saline mixtures of concentrations 0.6 to $2.0 \mathrm{mEq} / \mathrm{L}$ was pipetted into a test tube, then were diluted with water to make up to $1 \mathrm{~mL}$. About $100 \mu \mathrm{L}$ from this was then mixed with $100 \mu \mathrm{L}$ of $0.1 \mathrm{M} \mathrm{NaOH}, 10 \mu \mathrm{L}$ of $0.25 \mathrm{M} \mathrm{Na}_{2} \mathrm{CO}_{3}$, $40 \mu \mathrm{L}$ of water, $2.15 \mathrm{~mL}$ of $90 \%\left(\mathrm{CH}_{3}\right)_{2} \mathrm{SO}$, and $100 \mu \mathrm{L}$ of $1 \mathrm{mM}$ of Quinizarin in $\left(\mathrm{CH}_{3}\right)_{2} \mathrm{SO}$.

For testing of ultramicro volumes of blood (plasma) samples, the above procedure was modified. Instead, an amount of $500 \mu \mathrm{L}$ from spiked samples of plasma was pipetted into a test tube, then were diluted with water to make up to $1 \mathrm{~mL}$, then $50 \mu \mathrm{L}$ from diluted plasma was mixed with $100 \mu \mathrm{L}$ of $0.1 \mathrm{M} \mathrm{NaOH}, 10 \mu \mathrm{L}$ of $0.25 \mathrm{M} \mathrm{Na}_{2} \mathrm{CO}_{3}, 40 \mu \mathrm{L}$ of water, $2.15 \mathrm{~mL}$ of $90 \%\left(\mathrm{CH}_{3}\right)_{2} \mathrm{SO}$, and $50 \mu \mathrm{L}$ of $1 \mathrm{mM}$ of Quinizarin in $\left(\mathrm{CH}_{3}\right)_{2} \mathrm{SO}$.

\subsection{Instrument Settings and Data Analysis}

A Perkin Elmer Lambda 1050 dual-beam spectrophotometer (PerkinElmer Corp., Waltham, Massachusetts) was used with the TrayCell ${ }^{\mathrm{TM}}$ (Helma GmbH \& Co., Mulheim, Germany), a device that fits into the standard cuvette position on a spectrophotometer but comprises of prisms and fibers that serve to periscope the light beam up to the sampling window and then back to the detector of the instrument. In this way, the required sample volume is only 4 to $5 \mu \mathrm{L}$. The device includes a cap containing a mirror, which determines a constant measuring pathlength. In this case, a pathlength of $1.0 \mathrm{~mm}$ was used, and the reagent blank was measured separately then subtracted from the remainder of spectra.

Spectra were acquired in the range of 450 to $700 \mathrm{~nm}$ at increments of $1 \mathrm{~nm}$, and with a response time of $0.2 \mathrm{~s}$ and three running cycles for each sample. Slit width of the detector was fixed at $2 \mathrm{~nm}$, and both reference and sample attenuators were kept at $100 \%$. Preprocessing of acquired data included weighted least squares baseline correction, and Savitsky-Golay algorithms for both smoothing and second derivatives. Calibration models were produced using the multiple linear regression method using blood plasma samples of known spiked concentrations of lithium. The model was optimized by application of mean centering and internal cross validation (random subset). Data from blood plasma samples with unknown spiked lithium concentrations were used for validation.

The MATLAB ${ }^{\circledR}$ software (MathWorks Inc., Novi, Michigan), and the PLS-toolbox (Eigenvector Research Inc., Manson, Washington) MATLAB ${ }^{\circledR}$ add-on were used to perform spectral preprocessing and regression analysis.

\section{Results and Discussions}

The recorded FEP measurements of saline solutions, which were prepared to give $\mathrm{Li}+$ of 0.6 to $2.0 \mathrm{mmol} / \mathrm{L}$, are shown in Table 1 . These were carried out initially, and acquired readings were used to extrapolate molar lithium concentrations that were used as a reference. The corresponding spectral data is shown in Fig. 1. Similar to results reported previously, ${ }^{26}$ the spectral data of saline only solution, i.e., $0 \mathrm{mmol} / \mathrm{L} \mathrm{Li}+$, was subtracted from the remainder of collected spectra, which as a result showed intensity variations in absorption minima between 605 and $560 \mathrm{~nm}$ that correlate with the amounts of lithium in the sample, i.e., higher lithium concentrations in a sample expressed more intense absorptions.

As the bands of interest have been identified, tests were repeated on spiked samples of human blood plasma. Table 2 shows the FEP readings of prepared samples and the 
Table 1 Lithium concentrations in saline solutions.

$\mathrm{Li}+(\mathrm{mmol} / \mathrm{L})=$ FEP $(\mathrm{ppm}) / 6.6941$

\begin{tabular}{lcc}
\hline \multicolumn{3}{c}{ Baseline of FEP $(\mathrm{ppm})=10.4 / 6.6941=1.4983 \mathrm{Li}+(\mathrm{mmol} / \mathrm{L})$} \\
\hline Conc $(\mathrm{mEq} / \mathrm{L})$ & FEP $(\mathrm{ppm})$ & $\mathrm{Li}+(\mathrm{mmol} / \mathrm{L})$ \\
\hline 0.6 & 4.3 & 0.620 \\
0.8 & 5.4 & 0.778 \\
1.0 & 6.4 & 0.922 \\
1.2 & 7.5 & 1.081 \\
1.4 & 8.4 & 1.210 \\
1.6 & 9.6 & 1.383 \\
1.8 & 10.9 & 1.570 \\
2.0 & 12.3 & 1.772 \\
\hline
\end{tabular}

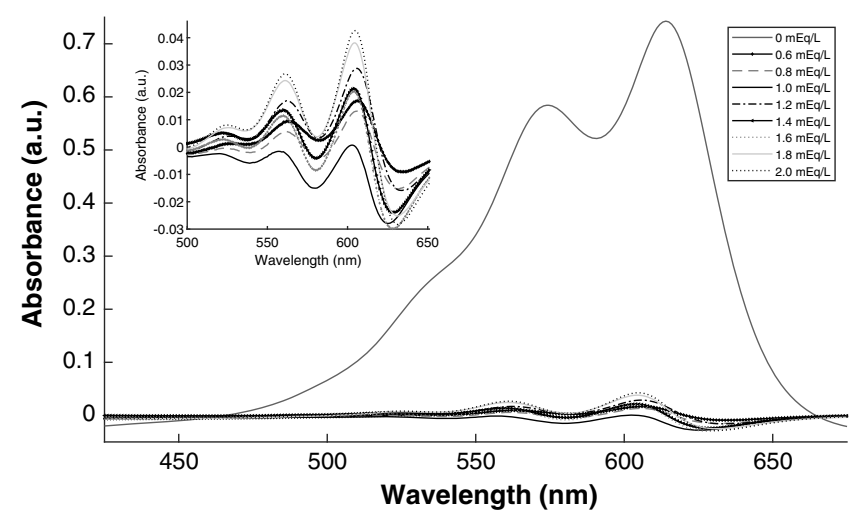

Fig. 1 Absorption spectra of saline-based solutions with lithium concentrations between 0.4 and $2.0 \mathrm{mmol} / \mathrm{L}$ in the range of 450 to $750 \mathrm{~nm}$.

Table 2 Lithium concentrations in blood plasma samples.

\begin{tabular}{lcc}
\hline \multicolumn{3}{c}{$\mathrm{Li}+(\mathrm{mmol} / \mathrm{L})=\mathrm{FEP}(\mathrm{ppm}) / 6.6941$} \\
\hline \multicolumn{3}{c}{ Baseline of FEP $(\mathrm{ppm})=10.4 / 6.6941=1.4983 \mathrm{Li}+(\mathrm{mmol} / \mathrm{L})$} \\
\hline Conc $(\mathrm{mEq} / \mathrm{L})$ & $\mathrm{FEP}(\mathrm{ppm})$ & $\mathrm{Li}+(\mathrm{mmol} / \mathrm{L})$ \\
\hline 0 & 0.6 & 0.086 \\
0.6 & 3.4 & 0.490 \\
0.8 & 4.2 & 0.605 \\
1.0 & 4.6 & 0.656 \\
1.2 & 5.5 & 0.792 \\
1.4 & 6.9 & 0.994 \\
1.6 & 7.6 & 1.095 \\
1.8 & 8.7 & 1.253 \\
2.0 & 9.5 & 1.369 \\
\hline
\end{tabular}

extrapolated molar concentrations of lithium, which were used as a reference in calibrations. Figure 2 shows the second derivative spectra of the same solutions. The spectral data of nonspiked blood plasma were used as a reference and subtracted from subsequent measurements. The figure shows a minima band around $604 \mathrm{~nm}$ that corresponds directly with lithium concentration, and a second minima around $555 \mathrm{~nm}$ expressing a less linear relationship with lithium levels. The regression calibration of spectral data against FEP measurements is shown in Fig. 3. The calibration was carried out using full-range spectra, i.e., 510 to $650 \mathrm{~nm}$, and after applying mean centering and random cross validation. The results indicated that a high correlation existed between the measured and predicted lithium values, with an $R^{2}$ value of 0.936588 and $\mathrm{RMSE}=0.0846769$. These results can also be seen in Table 3 .

As the development of a personal lithium monitor is one of the ultimate goals of this work, and that the use of full-range spectral data requires more complex data analysis methods, the possibility of isolating only two to three specific bands of interest was also explored. By specifying a limited number of wavelengths, simple and cheaper devices can be constructed. Based on the regression analysis of FEP and spectral measurements of spiked blood (plasma) samples, the selectivity ratio was examined. This graph, shown in Fig. 4, illustrates the

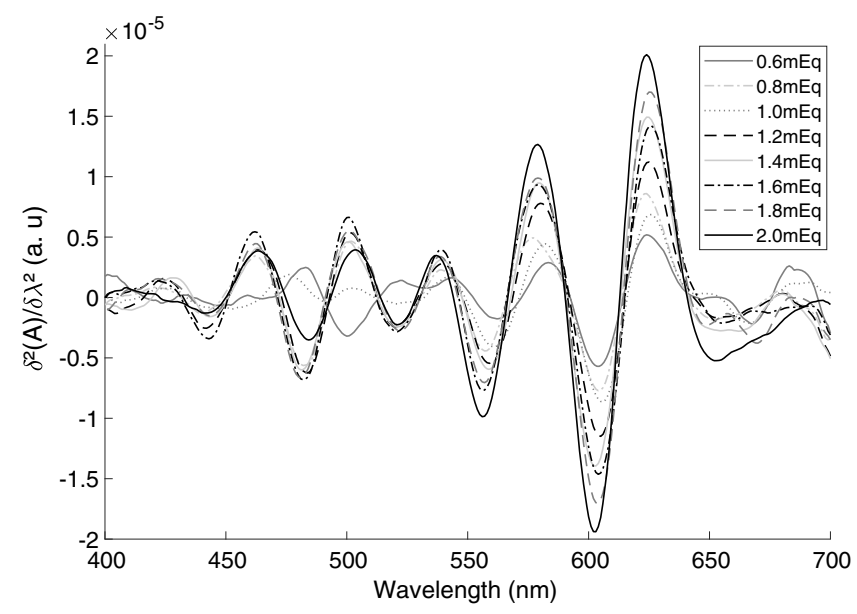

Fig. 2 Second derivative spectra of blood plasma-based solution of lithium concentrations between 0.6 and $2.0 \mathrm{mmol} / \mathrm{L}$.

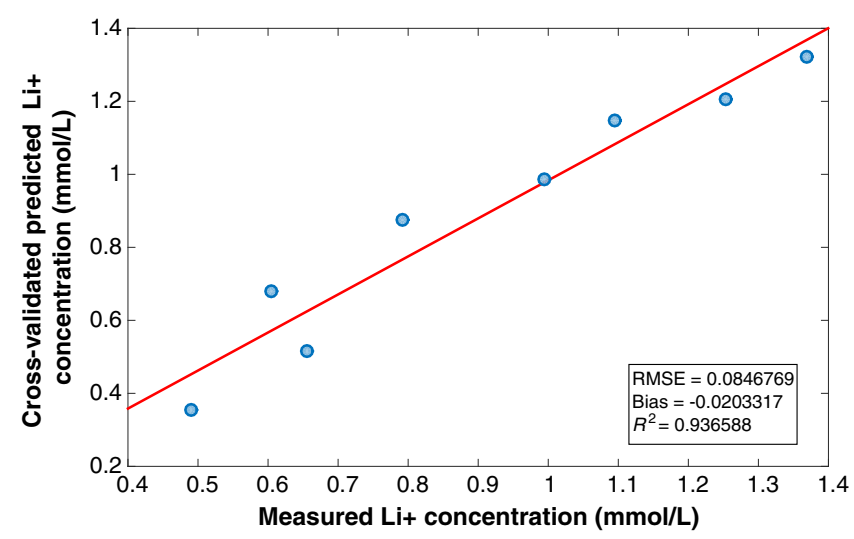

Fig. 3 Calibration curve of measured against predicted $\mathrm{Li}+$ values based on regression analysis of full-range spectra. 
Table 3 Calibration, cross-validation, and prediction results of regression analysis performed on full range spectra versus analysis on selected feature wavelengths for determination of lithium levels in blood plasma.

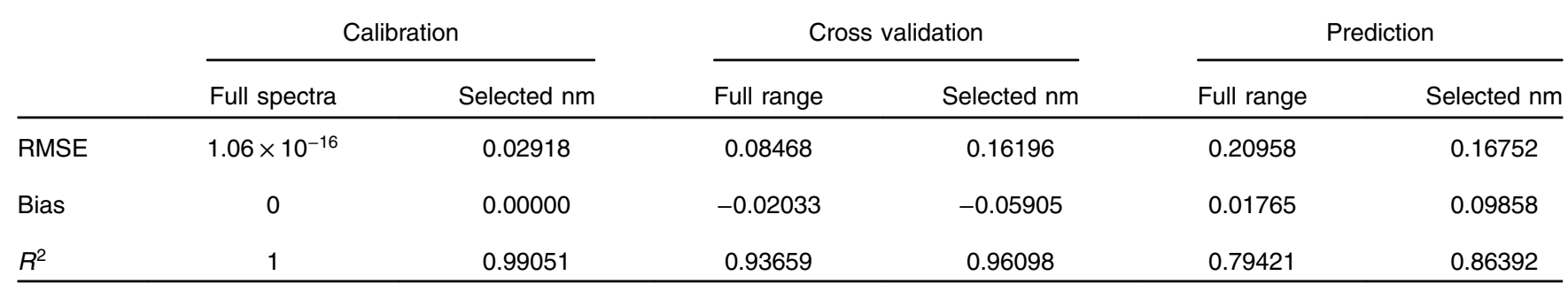

usefulness of each variable, i.e., wavelength and its contribution in the calculated regression. In this case, the results support the assumption that minima bands between 604 and $555 \mathrm{~nm}$ indicated significant variations in the spectra and hence were most useful toward explaining the calibration data. The focus here is on minima bands as in second derivative spectra, these correspond to absorption peaks in original spectra.

In addition to the two selected bands, a third at $522 \mathrm{~nm}$ was included as a reference. This point was selected as this region expressed the least contribution to the calibration, and hence, less susceptibility to variations in lithium concentrations.

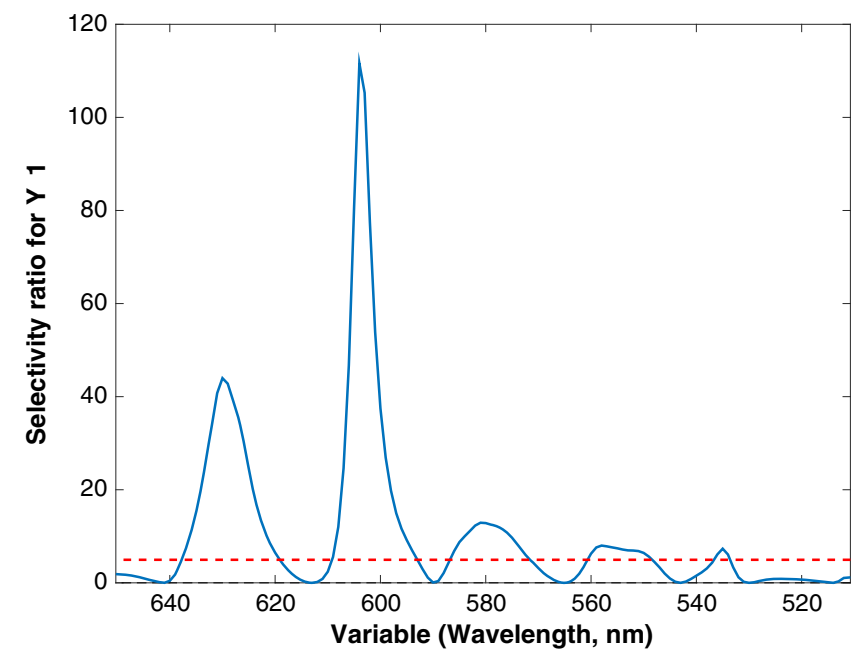

Fig. 4 Graph of selectivity ratio of $\mathrm{Li}+$ showing the main contributing bands to the regression model. $5 \%$ threshold.

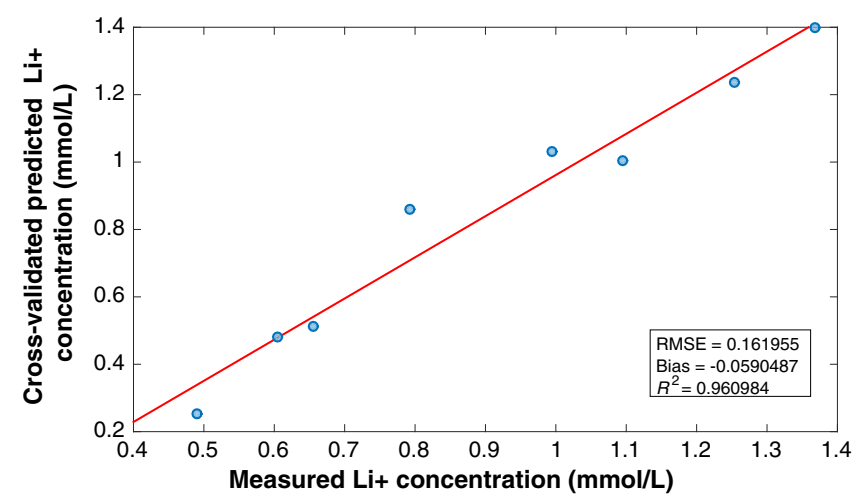

Fig. 5 Calibration curve of measured against predicted $\mathrm{Li}+$ values based on regression analysis of selected feature wavelengths.
Regression analysis was performed once again to verify the predictive ability of the selected feature wavelengths compared to that from full-range spectra. Input data were the average of points between 595 and $610 \mathrm{~nm}, 550$ and $570 \mathrm{~nm}, 514$ and $530 \mathrm{~nm}$, respectively. Figure 5 shows the cross-validated calibration of predicted lithium levels against measured values, and based on this, it is deduced that lithium concentrations can be predicted with good accuracy $\left(R^{2}=0.960984\right.$ and RMSE $=0.161955)$ using only three selected bands.

Furthermore, the validation of the generated calibration models was performed by testing a set of samples with unknown lithium concentrations. These samples were prepared as
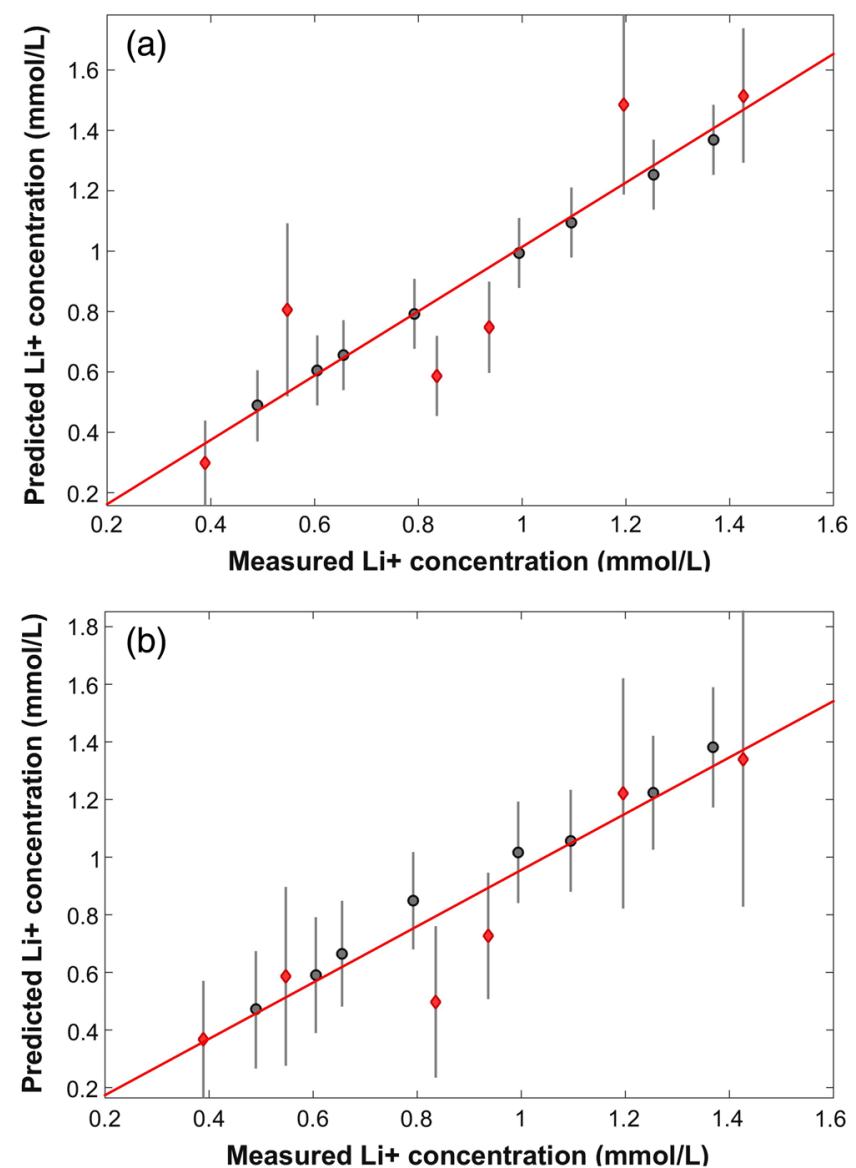

Fig. 6 Measured versus predicted values of blood (plasma) lithium concentrations estimated by regression analysis using (a) fullrange spectra and (b) selected feature wavelengths. Graphs include both calibration (gray) and predictive (red) values with their respective error. 
described in Sec. 2 and tested following the same procedure. Table 3 shows the prediction statistics and the performance of the regression.

It can be seen in Table 3 that although both methods had a good performance for predicting lithium levels, using only selected bands of interest improved the accuracy of prediction $\left(R^{2}=0.863921\right.$ compared with $\left.R^{2}=0.794214\right)$. This assumption is illustrated by Fig. 6, which shows a plot of the predicted concentrations of lithium resulting from both models including errors of each measurement. It is also evident that calibration and prediction results from full-range spectra are less erroneous than results from feature wavelengths, which although predicted lithium levels with more accuracy, presented greater deviations within each measurement.

\section{Conclusions}

An optical method that uses Quinizarin as a chromogenic agent was employed and modified to allow determinations of lithium levels in ultramicro volumes of blood plasma for applications in the management of bipolar mood disorder. Spectral variations in the regions between 604 and $555 \mathrm{~nm}$ were found to correspond linearly with lithium concentrations in spiked samples of human blood plasma, and regression analysis performed using fullrange spectra could predict lithium levels accurately. The selectivity ratio resulting from this analysis was used to select feature wavelengths. These were used as input to a second regression, which could also estimate lithium levels in samples of unknown concentration with improved accuracy, but with higher deviations between readings. Future work will focus on improving the predication ability of the calibration by testing against more unknown samples, as well as building and evaluating a miniaturized system, which would employ this measuring principle to provide readings of lithium levels in microvolumes of blood.

\section{Disclosures}

The authors have no relevant financial interests in this article and no potential conflicts of interest to disclose.

\section{Acknowledgments}

This report was independent research funded by the National Institute for Health Research (Invention for Innovation, Personal Lithium Blood Level Analyzer for patients with Bipolar Mood Disorder, II-LB-0313-20006). The views expressed in this publication are those of the author(s) and not necessarily those of the NHS, the National Institute for Health Research or the Department of Health.

\section{References}

1. S. Titmarsh, "The burden of bipolar disorder in the UK," Prog. Neurol. Psychiatry 16(5), 25-26 (2012).

2. J. R. Calabrese et al., "Impact of bipolar disorder on a U.S. community sample," J. Clin. Psychiatry 64(4), 425-432 (2003).

3. L. Fajutrao et al., "A systematic review of the evidence of the burden of bipolar disorder in Europe," Clin. Pract. Epidemiol. Ment. Health 5, 3 (2009).

4. A. Fagiolini et al., "Prevalence, chronicity, burden and borders of bipolar disorder," J. Affective Disord. 148(2-3), 161-169 (2013).

5. J. F. Hayes et al., "Lithium vs. valproate vs. olanzapine vs. quetiapine as maintenance monotherapy for bipolar disorder: a population-based UK cohort study using electronic health records," World Psychiatry 15(1), 53-58 (2016).
6. R. Oruch et al., "Lithium: a review of pharmacology, clinical uses, and toxicity," Eur. J. Pharmacol. 740, 464-473 (2014).

7. G. S. Malhi, "Safe and effective use of lithium," Aust. Prescr. 36(1), 18-21 (2013).

8. M. Sajatovic et al., "Treatment adherence with lithium and anticonvulsant medications among patients with bipolar disorder," Psychiatr. Serv. 58(6), 855-863 (2007).

9. N. Collins et al., "Standards of lithium monitoring in mental health trusts in the UK," BMC Psychiatry 10, 80 (2010).

10. A. McKean and J. Vella-Brincat, "Is it NICE to monitor lithium routinely?" N. Z. Med. J. 125(1355), 50-54 (2012).

11. M. Aliasgharpour and H. Hagani, "Evaluation of lithium determination in three analyzers: flame emission, flame atomic absorption spectroscopy and ion selective electrode," N. Am. J. Med. Sci. 1(5), 244-246 (2009).

12. A. Floris et al., "A prefilled, ready-to-use electrophoresis based lab-on-a-chip device for monitoring lithium in blood," Lab Chip 10(14), 1799-1806 (2010).

13. E. X. Vrouwe et al., "Microchip capillary electrophoresis for point-ofcare analysis of lithium," Clin. Chem. 53(1), 117-123 (2007).

14. D. Citterio et al., "pH-independent fluorescent chemosensor for highly selective lithium ion sensing," Anal. Chem. 79(3), 1237-1242 (2007).

15. C. A. Lindino et al., "Poly(o-methoxyaniline) modified electrode for detection of lithium ions," Quím. Nova 35(3), 449-453 (2012).

16. L. Constantinou et al., "On the merits of tetrapolar impedance spectroscopy for monitoring lithium concentration variations in human blood plasma," IEEE Trans. Biomed. Eng. 64, 601-609 (2016).

17. G. Dimeski, T. Badrick, and A. S. John, "Ion selective electrodes (ISEs) and interferences-a review," Clin. Chim. Acta 411(5-6), 309-317 (2010).

18. R. Luttge, Nano- and Microfabrication for Industrial and Biomedical Applications, William Andrew, Oxford (2016).

19. H. Whatley, "Basic principles and modes of capillary electrophoresis," in Clinical and Forensic Applications of Capillary Electrophoresis, J. R. Petersen and A. A. Mohammad, Eds., pp. 21-58, Humana Press, Totowa, New Jersey (2001).

20. J. P. Schaeper and M. J. Sepaniak, "Parameters affecting reproducibility in capillary electrophoresis," Electrophoresis 21(7), 1421-1429 (2000).

21. L. C. Rodríguez, C. J. Linares, and M. R. Ceba, "Specific fluorescence detection of lithium ion using quinizarin and extraction in TBP," Anal. Lett. 27(8), 1569-1577 (1994).

22. L. C. Rodriguez, C. J. Linares, and M. R. Ceba, "Selective spectrofluorometric determination of lithium(I) with quinizarin by extraction into tributyl phosphate," Fresenius J. Anal. Chem. 356(5), 320-325 (1996).

23. J. H. Kim, D. Diamond, and K. T. Lau, "Development of portable device for monitoring the lithium level from bipolar disorder patients," in 5th Int. Conf. on Pervasive Computing Technologies for Healthcare (PervasiveHealth) and Workshops, pp. 230-233 (2011).

24. L. G. Gracia, L. C. Rodríguez, and M. R. Ceba, "Spectrophotometric determination of lithium with Quinizarin in drugs and serum," Talanta 44(1), 75-83 (1997).

25. M. Tabata, J. Nishimoto, and T. Kusano, "Spectrophotometric determination of lithium ion using a water-soluble octabromoporphyrin in aqueous solution," Talanta 46(4), 703-709 (1998).

26. M. Qassem, M. Hickey, and P. A. Kyriacou, "Colorimetric determinations of lithium levels in drop-volumes of human plasma for monitoring patients with bipolar mood disorder," in 38th Annual Int. Conf. of the IEEE Engineering in Medicine and Biology Society (EMBC), pp. 51605163 (2016).

Meha Qassem is a postdoctoral research associate in the Research Centre for Biomedical Engineering (RCBE) at City University of London. Her research interests are focused on the areas of optical spectroscopy, biosensing, and medical electronics. She has worked extensively in the area of near-infrared (NIR) spectroscopy and has successfully completed multiple animal and human studies to study the properties of skin, mental health, and hemodynamic shock in critically ill patients.

lasonas Triantis is a senior lecturer in microelectronics and bioinstrumentation at the Research Centre for Biomedical Engineering, City 
University London, London, UK. His main research activities are focused on bioimpedance and neural interfacing diagnostic and therapeutic devices. He has previously worked on the design of implantable neuroprostheses, as well as devices and systems for electrical impedance tomography and bio-impedance measurements with applications in blood analysis, blood flow, and bowel cancer detection.

Michelle Hickey was awarded a $\mathrm{PhD}$ in biomedical engineering from City University London in January 2010. She has many years of experience in developing electro-optical and fiber optic sensors, and designing and implementing medical electronics. Her research interests include areas such as tissue optics, sensors, medical electronics, and cardiovascular measurement.

Eleni Palazidou (MD, PhD, MRCP, FRCPSYCH) is a consultant psychiatrist with particular expertise in the assessment and treatment of
Mood Disorders and in Psychopharmacology. She trained as a psychiatrist at the Maudsley Hospital and did her $\mathrm{PhD}$ on the mechanism of action of antidepressant drugs at the Institute of Psychiatry. She has been involved in research and teaching for many years. Currently, she involved mainly in academic activities as a professor at the University of Nicosia Medical School and honorary professor in psychiatry at Barts \& London School of Medicine.

Panayiotis A. Kyriacou is a professor of biomedical engineering associate dean for Research and Enterprise at City University London, and director of the Biomedical Engineering Research Centre. His main research activities are primarily focused upon the understanding, development, and applications of instrumentation, sensors, and physiological measurement to facilitate the prognosis, diagnosis, and treatment of disease or the rehabilitation of patients. 